$13^{\text {th }}$ International Conference on AEROSPACE SCIENCES \& AVIATION TECHNOLOGY, ASAT-13, May 26 - 28, 2009, E-Mail: asat@mtc.edu.eg Military Technical College, Kobry Elkobbah, Cairo, Egypt Tel : +(202) $24025292-24036138$, Fax: +(202) 22621908

\title{
Numerical Aerodynamic Characteristics of Flapping Wings
}

\author{
M. Y. Zakaria , A. M. Elshabka* ${ }^{* *}$ A. M. Bayoumy $^{* *}$, O. E. Abd Elhamid ${ }^{* * *}$
}

\begin{abstract}
The unsteady aerodynamic characteristics of a flapping wing are studied. The computational model for the unsteady aerodynamics of flapping wing using strip theory approach has been developed and clarified. The proposed method is used to solve the mechanical flying ornithopter (SlowHawk 2) of flexible wing membrane. In doing so, the model is verified through the computations performed on a mechanical flying Pterosaur replica as well as smaller biological species including the Corvus monedula and Larus canus. The effect of aerodynamic parameters on the performance of these biological flight vehicles is studied. The results are compared with those available in the literature, the overall agreement is excellent. The effect of Reduced frequency is studied defining an optimal design points for sustainable flight conditions ( $\bar{L}>\mathrm{W})$. A manual optimization is performed on the developed code for the SlowHawk 2 in order to get predicted values to be used as an input data for calculating the optimum aerodynamic characteristics of it.
\end{abstract}

Keywords: Unsteady Aerodynamics, Flapping Wing and Reduced frequency

\section{Introduction}

Among all means of propulsion for low Reynolds number flight, flapping wings is the highly efficient one, as evidenced by the abundant examples we observe in nature. However the aerodynamics of flapping flight is not fully understood. The motivation for this work is based on the challenges of modeling a biological flapping wing, their wing design is complex and having flexible nature. Previous works show that there are two main models for analyzing the unsteady flow condition encountered during flapping wing flight, which are the quasi-steady model and the wake model. In the quasi-steady model, the unsteady wake effects are ignored. (i.e. That is, flapping frequencies are assumed to be slow enough that shed wake effect are negligible). This may lead to a model being unsuitable for cases of high flapping frequencies where the shed wake effects cannot be ignored. Although such an assumption gives a great simplification to the aerodynamic modeling, this category can still contain a wide range of sophistication in its detailed approaches.

The computation performed in this work tries to address these issues. In doing so, the used kinamatic model is based on the model proposed by Delaurier [1], which is based on strip theory and used for predicting average Lift and Thrust for pterosaur replica. The wing is assumed to be rigid in the spanwise direction, this is suitable for wings with high aspect-ratio.

\footnotetext{
Egyptian Armed Forces, Email: $\underline{\text { m_y_zakaria@hotmail.com }}$

** Egyptian Armed Forces

${ }^{* * *}$ Professor, Aeronautical Engineering
} 
A continuous harmonic sinusoidal motion is assumed. So there is equal time for upstroke and downstroke, this is a simplifying assumption because in real biological flight the downstroke time is more than upstroke time. It also accounts for camber and leading edge suction effects, which is a phenomena associated with biological wings. Post-stall behavior is also accounted for in this model. With this theory, wing bending can be accounted for in the kinematic model, although bending effects are ignored in this particular model. The relative angle of attack will not fall below zero (no negative stalling), consequently the minimum stall angle of attack will not be specified. When the time dependence is included, the flow conditions for each strip will change in time, and hence the change in Lift and Thrust generated. The total Lift and Thrust for the wing is calculated by the summation of the contributions from each segment for a whole flapping cycle. Daniel et al. [2] studied the effect of aerodynamic parameters based on Delaurier model the performance of a mechanical flying pterosaur replica as well as smaller biological species including Corvus monedula, Larus Canus and Columba livia, A design of an efficient Ornithopter wing was introduced by Bendict [3], using aeroelastic modeling and strip theory.

\section{Mathematical Formulation}

The wing kinematics, forces, power and propulsive efficiency calculation are presented, as well as the design points limited by the reduced frequency range of sustained flight regime are also presented.

\subsection{Flapping wing Kinematics}

The wing kinematics and wing sections are illustrated in Fig.1.

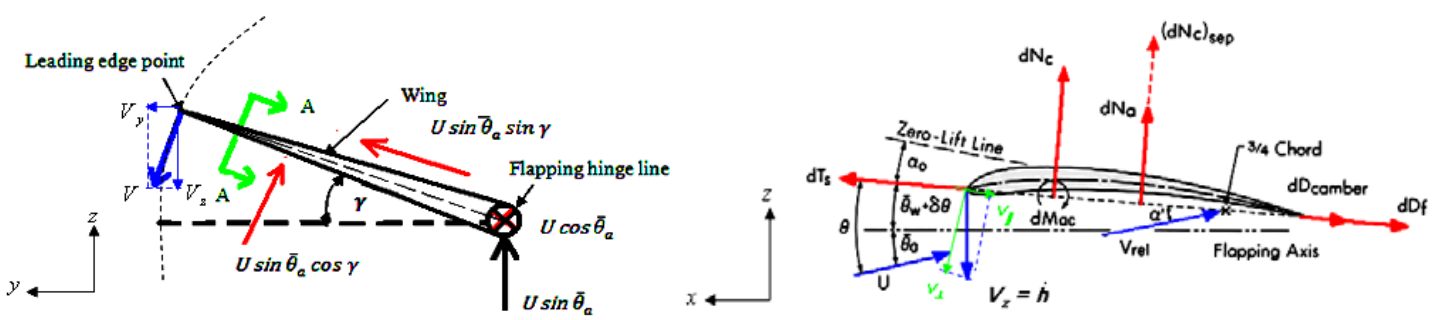

Fig. 1 A front and section view of flapping wing

For a root flapping motion with no spanwise bending, as a result, the plunging motion is given by:

$$
h=-(\Gamma y) \sin (\phi)
$$

where $h \quad$ is the plunging displacement

$\Gamma \quad$ is the maximum flapping angle

$\phi \quad$ is the cycle angle, defined by $(\omega t)$

$\omega \quad$ is the angular flapping frequency in $\mathrm{rad} / \mathrm{s}$

The dynamic twist is linearly proportional to the span, according to the following relation:

$$
\delta \theta=-\left(\beta_{O} y\right) \cos (\phi)
$$

where $\quad \delta \theta \quad$ is the dynamic varying pitch angle and is given by: 


$$
\delta \theta=(\theta-\bar{\theta})
$$

where $\quad \begin{array}{r}\theta \\ \theta\end{array}$ is the pitch angle of airfoil chord with respect to free stream velocity $U$.

$\bar{\theta} \quad$ is the section's mean pitch angle, and is given by:

$$
\bar{\theta}=\bar{\theta}_{a}+\bar{\theta}_{w}
$$

where $\quad \bar{\theta}_{a} \quad$ is the pitch angle of flapping axis with respect to $U$

$\bar{\theta}_{w} \quad$ is the mean pitch angle of chord with respect to flapping axis

In the case of whole wing motion (no flapping axis), $\bar{\theta}$ will be the wing's mean pitch angle. Upon using the leading edge as the reference point, the wing's motion consists of three discrete motions, these motions are the plunging motion, pitching motion and the forward motion relative to the freestream velocity $(U)$. The component of plunging velocity $\dot{h}$ in a direction perpendicular to the airfoil chord line seen at each instant of time is $\dot{h} \cos \left(\theta-\bar{\theta}_{a}\right)$. In pitching motion, the $3 / 4$ chord point is the point of concern, and then the radius of rotation $3 / 4$ chord giving a rotational velocity equals $3 / 4 \mathrm{c} \dot{\theta}$. Finally in forward motion, the instantaneous relative angle of attach $\delta \theta$ giving a velocity in vertical direction $U \delta \theta$, substituting in equation (3) giving the velocity equals $U(\theta-\bar{\theta})$. As a result of the wing motion discussed above, we can easily calculate the relative angle of attach $\alpha$ at $3 / 4$ chord location due to wing's motion as:

$$
\alpha=\frac{\left(\dot{h} \cos \left(\theta-\bar{\theta}_{a}\right)+\frac{3}{4} C \dot{\theta}+U(\theta-\bar{\theta})\right)}{U}
$$

\subsection{Force Calculation}

For calculation of the forces generated during a flapping cycle, the flow relative velocity and flow relative angle of attack should be determined first, using the derived expression of relative angle of attack at $3 / 4$ chord location due to wing's motion $\alpha$ given by equation (5), the flow's relative angle of attach $\alpha^{\prime}$ at $3 / 4$ chord location is given by:

$$
\alpha^{\prime}=\left[C(k)_{\text {Jones }}\right] \alpha-\frac{W_{o}}{U}
$$

where $\quad W_{o} \quad$ is the downwash velocity at $3 / 4$ chord location The coefficient of $\alpha^{\prime}$ in equation (6) is derived by Jones [4] accounts for the wing's finite span unsteady vortex wake by means of strip theory model. He uses modified Theodorsen function for finite aspect ratio wings and is given by:

$$
C(k)_{\text {Jones }}=\left(\frac{\lambda}{2+\lambda}\right) C^{\prime}(k)
$$

where $\quad \lambda$ is the wing aspect ratio

$k \quad$ is the reduced frequency given by: 


$$
k=\frac{c \omega}{2 U}
$$

$C(k)_{\text {Jones }}$ is a complex function, and it was found convenient to use Scherer's [5] alternative formulation where the complex form of $C^{\prime}(k)$ is given by:

$$
C^{\prime}(k)=F^{\prime}(k)+i G^{\prime}(k)
$$

Substituting equation (9) into equation (7), Then substituting equation (7) into equation (6) with the assumed motion given by:

$$
\alpha=A e^{-w t}
$$

We obtain an expression for $\alpha^{\prime}$ as:

$$
\alpha^{\prime}=\frac{\lambda}{(2+\lambda)}\left[F^{\prime}(k) \alpha+\frac{C}{2 U} \frac{G^{\prime}(k)}{k} \dot{\alpha}\right]-\frac{W_{o}}{U}
$$

where $\quad \frac{W_{o}}{U} \quad$ is the downwash term and is given by:

$$
\frac{W_{o}}{U}=2 \frac{\left(\alpha^{\prime}+\bar{\theta}\right)}{(2+\lambda)}
$$

\subsubsection{Normal force calculation}

The normal force calculation on the wing differs depending on whether the flow is attached or separated. Some modifications have to be made to treat the separated flow condition. To calculate the Normal force for attached flow, the section's normal force due to circulation is given by:

$$
d N_{c}=\frac{1}{2} \rho U V C_{n}(y) c d y
$$

where, the normal force coefficient is given by:

$$
C_{n}(y)=2 \pi\left(\alpha^{\prime}+\alpha_{o}+\bar{\theta}\right)
$$

where $\quad \alpha_{o} \quad$ is the airfoil zero Lift angle

Another additional Normal force contribution comes from the apparent mass effect or virtual mass force. The acceleration of this mass of air shows up as a virtual mass force which acts at the mid-chord and is given by:

$$
d N_{a}=\frac{\rho \pi c^{2}}{4} \dot{v}_{2} d y
$$


where $\rho \pi c^{2} / 4$ is the mass of air enclosed in a thin cylinder of width $d y$ and of diameter equal to the chord.

$\dot{v}_{2} \quad$ is the time rate of change of the mid-chord normal velocity component due to the wing's motion and is given by:

$$
\dot{v}_{2}=U \dot{\alpha}-\frac{1}{4} c \ddot{\theta}
$$

Therefore, the section's total attached flow normal force is

$$
d N=d N_{a}+d N_{c}
$$

For the Normal force calculation for separated flow, some modifications have to be made for the case when the flow is separated. As the strip theory model allows for an approximation to localized post stall behavior, the stall behavior is strictly dependant on the static stall characteristics for the airfoil, which is defines as follows:

$$
\left(\alpha_{\text {stall }}\right)_{\min } \leq\left[\alpha^{\prime}+\bar{\theta}-\frac{3}{4}\left(\frac{c \dot{\theta}}{U}\right)\right] \leq\left(\alpha_{\text {stall }}\right)_{\max }
$$

Since, it is assumed that negative $\alpha^{\prime}$ stalling would not occur, hence the lower limit is of no interest. When the upper limit is exceeded, the flow is separated and the normal force under separated condition is written as:

$$
d N=\left(d N_{c}\right)_{\text {sep }}+\left(d N_{a}\right)_{\text {sep }}
$$

where $\left(d N_{c}\right)_{\text {sep }}$

is due to crossflow drag and is given by:

$$
\left(d N_{c}\right)_{\text {sep }}=\left(C_{d}\right)_{c f} \frac{1}{2} \rho \widehat{V} V_{n} c d y
$$

where $\left(C_{d}\right)_{c f} \quad$ is the post stall normal force coefficient and according to Hoerner [6], its value is 1.98 .

$\widehat{V}$ is the resultant of the chordwise component and is given by:

$$
\sqrt{\left(V_{x}^{2}+V_{n}^{2}\right)}
$$

Also, $\left(d N_{a}\right)_{\text {sep }}$ due to apparent mass effects is assumed to be half that of the attached flow value in equation (15) and is given by:

$$
\left(d N_{a}\right)_{\text {sep }}=\frac{1}{2}\left(d N_{a}\right)
$$

\subsubsection{Chordwise force calculation}

The section's circulation distribution likewise generates forces in the chordwise direction, as illustrated in Fig.1. The chordwise force due to camber is given by: 


$$
d D_{\text {camber }}=-2 \pi \alpha_{o}\left(\alpha^{\prime}+\bar{\theta}\right) \frac{1}{2} \rho U V c d y
$$

Garrick [7] presented a theory where the leading-edge suction is examined for a twodimensional airfoil. Incorporating his theory to the strip theory model, we get an expression for the chordwise force due to leading-edge suction, which reads as:

$$
d T_{s}=\eta_{s} 2 \pi\left(\alpha^{\prime}+\bar{\theta}-\frac{1}{4} \frac{c \dot{\theta}}{U}\right)^{2} \frac{1}{2} \rho U V c d y
$$

where $\quad \eta_{s} \quad$ is the leading edge suction efficiency factor

Viscosity also gives a chordwise friction drag as:

$$
d D_{f}=\left(C_{d}\right)_{f} \frac{1}{2} \rho V_{x} c d y
$$

where $\quad\left(C_{d}\right)_{f} \quad$ is the drag coefficient due to skin friction.

Thus, the total chordwise force is given by:

$$
d F_{x}=d T_{s}-d D_{\text {camber }}-d D_{f}
$$

When the attached flow range is exceeded, totally separated flow is assumed to abruptly occur. For that condition, all chordwise forces are negligible.

\subsubsection{Lift and Thrust}

Now, the equations for the segment's instantaneous Lift and Thrust are:

$$
\begin{aligned}
& d L=d N \cos \theta+d F_{x} \sin \theta \\
& d T=d F_{x} \cos \theta-d N \sin \theta
\end{aligned}
$$

These may be integrated along the span to give the whole wing's instantaneous Lift and Thrust for the whole wing as:

$$
L(t)=2 \int_{0}^{\frac{b}{2}} \cos (\gamma(t)) d L
$$

where $\quad \gamma(t) \quad$ is the section's dihedral angle at that instant in the flapping cycle

$$
T(t)=2 \int_{0}^{\frac{b}{2}} d T
$$

The wing's average Lift and Thrust are obtained by integrating $L(t)$ and $T(t)$ over the cycle. 


$$
\begin{aligned}
& \bar{L}=\frac{1}{2 \pi} \int_{0}^{2 \pi} L(\phi) d \phi \\
& \bar{T}=\frac{1}{2 \pi} \int_{0}^{2 \pi} T(\phi) d \phi
\end{aligned}
$$

\subsubsection{Power and Propulsive efficiency}

For attached flow the instantaneous power required to move the section against its aerodynamic loads is given by:

$$
\begin{aligned}
d P_{i n}= & d F_{x} \dot{h} \sin \left(\theta-\bar{\theta}_{a}\right)+d N\left[\dot{h} \cos \left(\theta-\bar{\theta}_{a}\right)+\frac{1}{4} c \dot{\theta}\right]+d N_{a}\left[\frac{1}{4} c \dot{\theta}\right] \\
& -d M_{a c} \dot{\theta}-d M_{a} \dot{\theta}
\end{aligned}
$$

where $\quad d M_{a c} \quad$ is the section's pitching moment about its aerodynamic centre and depends on the airfoil characteristics

$d M_{a} \quad$ Includes apparent-camber and apparent-inertia moments and given by:

$$
d M_{a}=-\left[\frac{1}{16} \rho \pi c^{3} \dot{\theta} U+\frac{1}{128} \rho \pi c^{4} \ddot{\theta}\right] d y
$$

For separated flow, equation (33) is simplified by ignoring $d F_{x}, d M_{a c}$ and $d M_{a}$, so we have:

$$
d P_{\text {in }}=d N_{\text {sep }}\left[\dot{h} \cos \left(\theta-\bar{\theta}_{a}\right)+\frac{1}{2} c \dot{\theta}\right]
$$

The instantaneous aerodynamic power absorbed by the whole wing is found from the integration all over the span, and the average input power throughout the cycle is obtained by the integration all over the flapping cycle $(\phi)$. Since the flight speed $U$ is constant, we can determine the average output power by multiplying the average Thrust with the flight speed, so we have:

$$
\bar{P}_{\text {out }}=\overline{T U}
$$

Therefore, the average propulsive efficiency may be calculated from:

$$
\bar{\eta}=\frac{\bar{P}_{\text {out }}}{\bar{P}_{\text {in }}}
$$

\subsection{Computational procedures}

This section will cover descritization of the equations forming the flapping wing aerodynamic model followed by step by step computational procedures as well as, optimization procedures in order to predict the values of aerodynamic parameters needed for measuring other species. The airfoil used is Liebeck LPT $110 \mathrm{~A}$ for all the test cases. The details of the airfoil are given in Table 1.. 
Table 1 Aerodynamic data for Liebeck $L P T$ airfoil

\begin{tabular}{|c|c|c|}
\hline Symbol & Value & Description \\
\hline$\alpha_{o}$ & $0.5[\mathrm{Deg}]$ & Angle of sections zero Lift line \\
\hline$\eta_{s}$ & 0.98 & Leading edge suction efficiency \\
\hline$C_{\text {mac }}$ & 0.025 & Moment coefficient about aerodynamic centre \\
\hline$\left(\alpha_{\text {stall }}\right)_{\max }$ & $13[\mathrm{Deg}]$ & Airfoil’s stall angle \\
\hline
\end{tabular}

There are three different programs to determine the different parameters considered during the flapping cycle. The first of them performs calculations for Pterosaur [8], which was presented by Delaurier [1] in order to verify the MATLAB code. The second program is developed to determine the design points from operating reduced frequency for sustained flight [8].

A parametric optimization study is performed with variable undisturbed flow $U$ and fixing the flapping frequency $f$, in order to have a change in the measured reduced frequency. This helps us for predicting their design point margin. Finally, a manual optimization program is developed [8] to get suitable input aerodynamic parameters for the unsteady code of the SlowHawk 2 ornithopter. This is done with the help of the optimization results presented by Daniel [2] in order to have an optimum input parameters such as the dynamic twist $\left(\beta_{o}\right)$ and flapping frequency $(f)$.

Table 2 Input data for tested models

\begin{tabular}{|c|c|c|c|c|c|c|c|}
\hline Test Model & $\mathbf{W}(\mathbf{k g})$ & $\mathbf{b}(\mathbf{m})$ & $\mathbf{f}(\mathbf{H z})$ & $\mathbf{U}(\mathbf{m} / \mathbf{s})$ & $\Gamma(\mathbf{D e g})$ & $\bar{\theta}_{a}(\mathbf{D e g})$ & $\beta_{o}(\mathbf{D e g} / \mathbf{m})$ \\
\hline Pterosaur & 18.14 & 5.48 & 1.2 & 13.411 & 20 & 7.5 & $0-10$ \\
\hline Jack Daw & 0.171 & 0.544 & 5.75 & 11 & 70 & 7.4 & $0-165$ \\
\hline Mew Gull & 0.3717 & 1.12 & 3.5 & 9.2 & 35 & 5.8 & $0-65$ \\
\hline SlowHawk 2 & 0.42 & 1.22 & $2.8-3.6$ & $5-8$ & $25 \pm 10$ & $4.2-5.2$ & $0-70$ \\
\hline
\end{tabular}

\subsubsection{Pterosaur replica}

The wing is divided into twelve strips shown in Table 3. of equal width $(d y=0.224 \mathrm{~m})$. Also 20 time step interval were chosen.

Table 3 Mean chord of the twelve strips for pterosaur

\begin{tabular}{|c|c|c|c|c|c|c|c|c|c|c|c|c|}
\hline Section No. & 1 & 2 & 3 & 4 & 5 & 6 & 7 & 8 & 9 & 10 & 11 & 12 \\
\hline Chord (m) & 0.744 & 0.607 & 0.515 & 0.452 & 0.416 & 0.411 & 0.424 & 0.363 & 0.309 & 0.289 & 0.231 & 0.127 \\
\hline
\end{tabular}

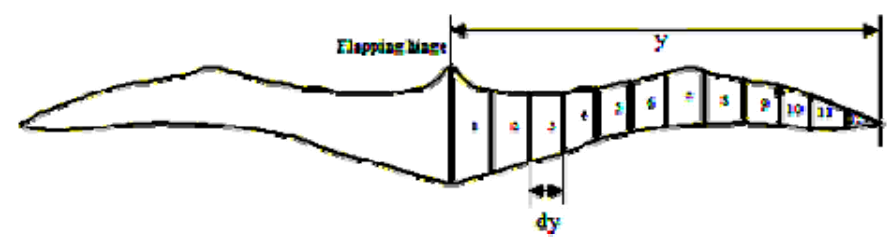

Fig. 2 Schematic diagram of a Pterosaur Replica wing 
The results we have obtained shows close correlation with the results obtained by Delaurier [1], Daniel [2] and Benedict [3]. For the given flapping frequency of $1.2 \mathrm{~Hz}$, it is seen that the wings will Lift the model's 18.478 weight if the flapping-axis angle $\bar{\theta}_{a}$ equals $7.5^{\circ}$, the flight speed $\mathrm{U}$ equals $13.4 \mathrm{~m} / \mathrm{s}$, and the dynamic twist magnitudes $\beta_{o}$ are in excess of $7.3(\mathrm{deg} / \mathrm{m})$.
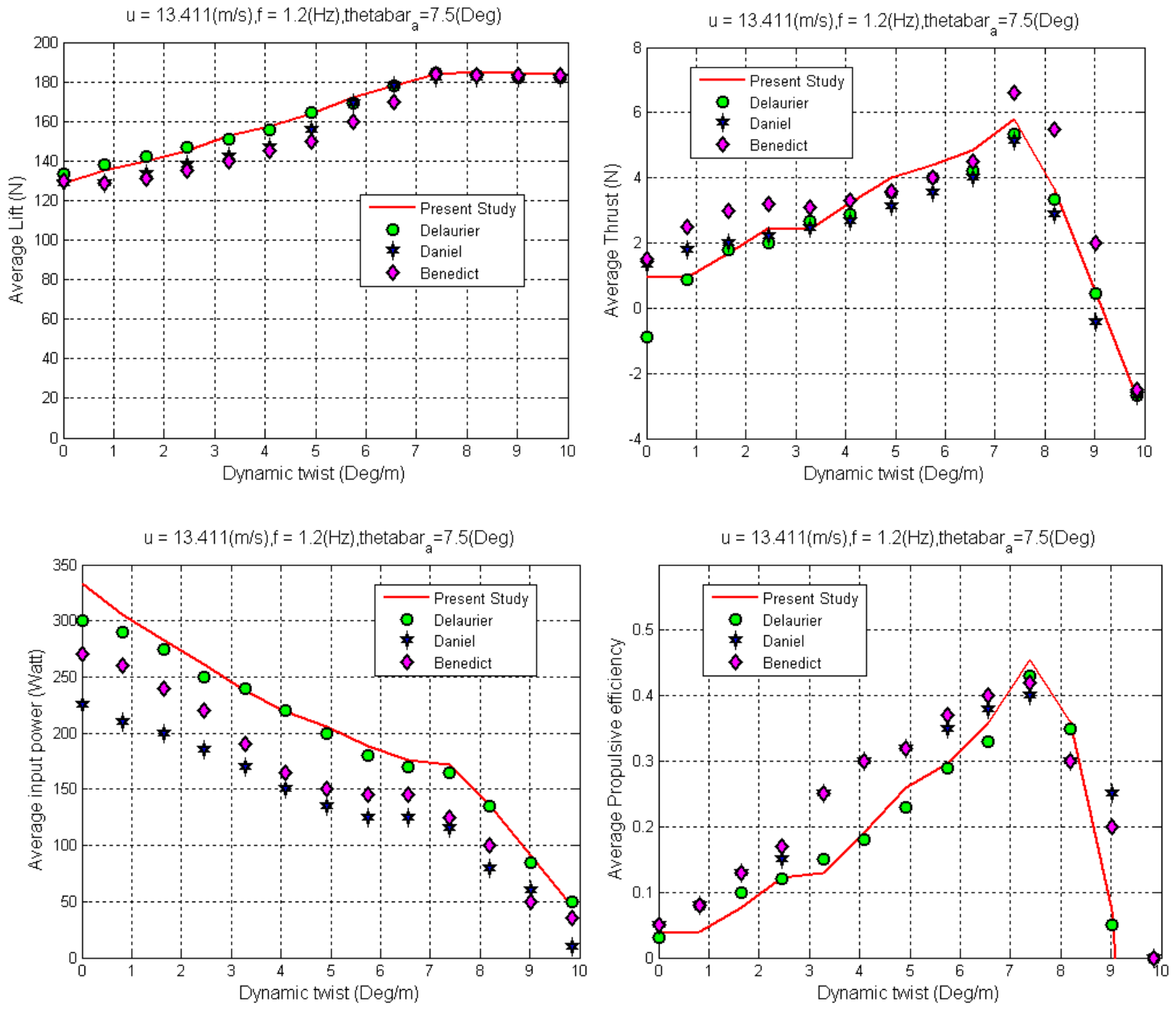

Fig. 3 Pterosaur replica results

To introduce the effect of reduced frequency variation $k$ on the aerodynamic performance, we must choose a given chord to deal with it. As the bird species can be compared to each other by the value of their optimum value of reduced frequency, we can choose the mean chord length along the span for each case study. Since the reduced frequency by definition has two operating variables $f$ and $U$, in this analysis we fix the flapping frequency $(f)$ and taking various values for freestreem from $U=11-14(\mathrm{~m} / \mathrm{s})$ giving a reduced frequency margin from $k=0.1-0.139$. The results of this analysis are shown in Fig. 4.

From Fig.4., it is clear that for increasing the value of reduced frequency the average lift decreases, ( $L \propto U^{2}$ ). The average thrust and propulsive efficiency, till a certain value of dynamic twist (5.7 deg/m), is directly proportional to the reduced frequency. As by increasing $\mathrm{k}$, the average Thrust increases. After the inflection value, the average Thrust and Propulsive efficiency starts to be inversely proportional with the reduced frequency. It is seen that the input power is inversely proportional with the reduced frequency. Increasing the reduced frequency $(k)$, the average input power changes its behavior relative to an inflection point at 
dynamic twist equals ( $8 \mathrm{deg} / \mathrm{m})$. It is clear that, the design point for obtaining the maximum propulsive efficiency and optimum aerodynamic performance locating between the two inflection points at dynamic twist values $5.7(\mathrm{deg} / \mathrm{m})$ and $8(\mathrm{deg} / \mathrm{m})$.

\subsubsection{Corvus monedula (Jack Daw) and Mew Gull}

The results obtained for Jack Daw and Larus Canus are presented in Fig. 5. and Fig. 6. respectively. Also it is clear the excellent agreement with the previous work by Daniel [2] and Benedict [3].

From the analysis above, the value of the dynamic twist is considerably higher for the Corvus monedula than for the Pterosaur. This shows that a long wing can have a smaller dynamic twist than a shorter one and still have enough twist to generate sufficient thrust.

The obtained aerodynamic characteristics values are considered to be a predicted input values for our optimization trials.
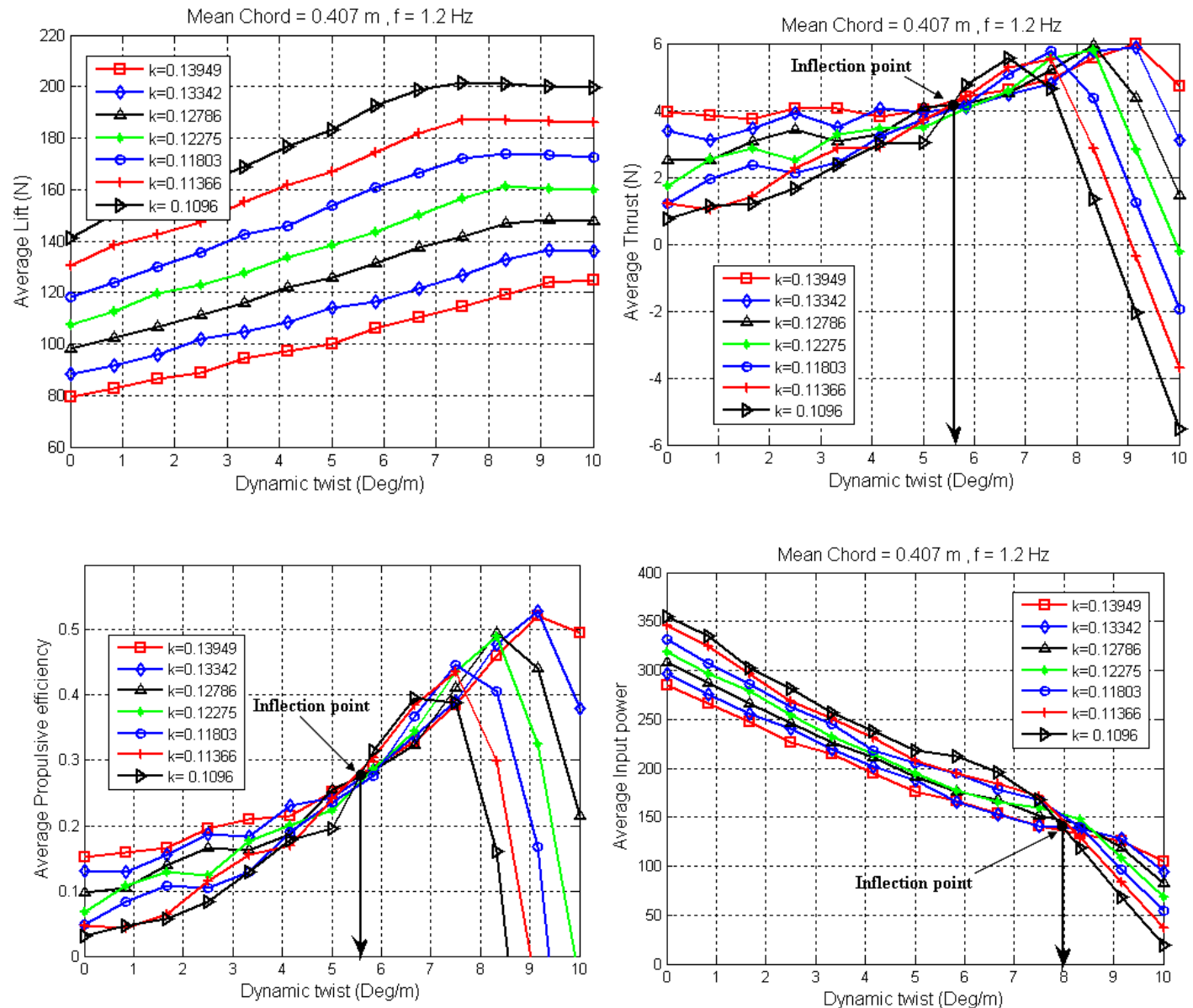

Fig. 4 Reduced frequency variation vs. aerodynamic parameters 

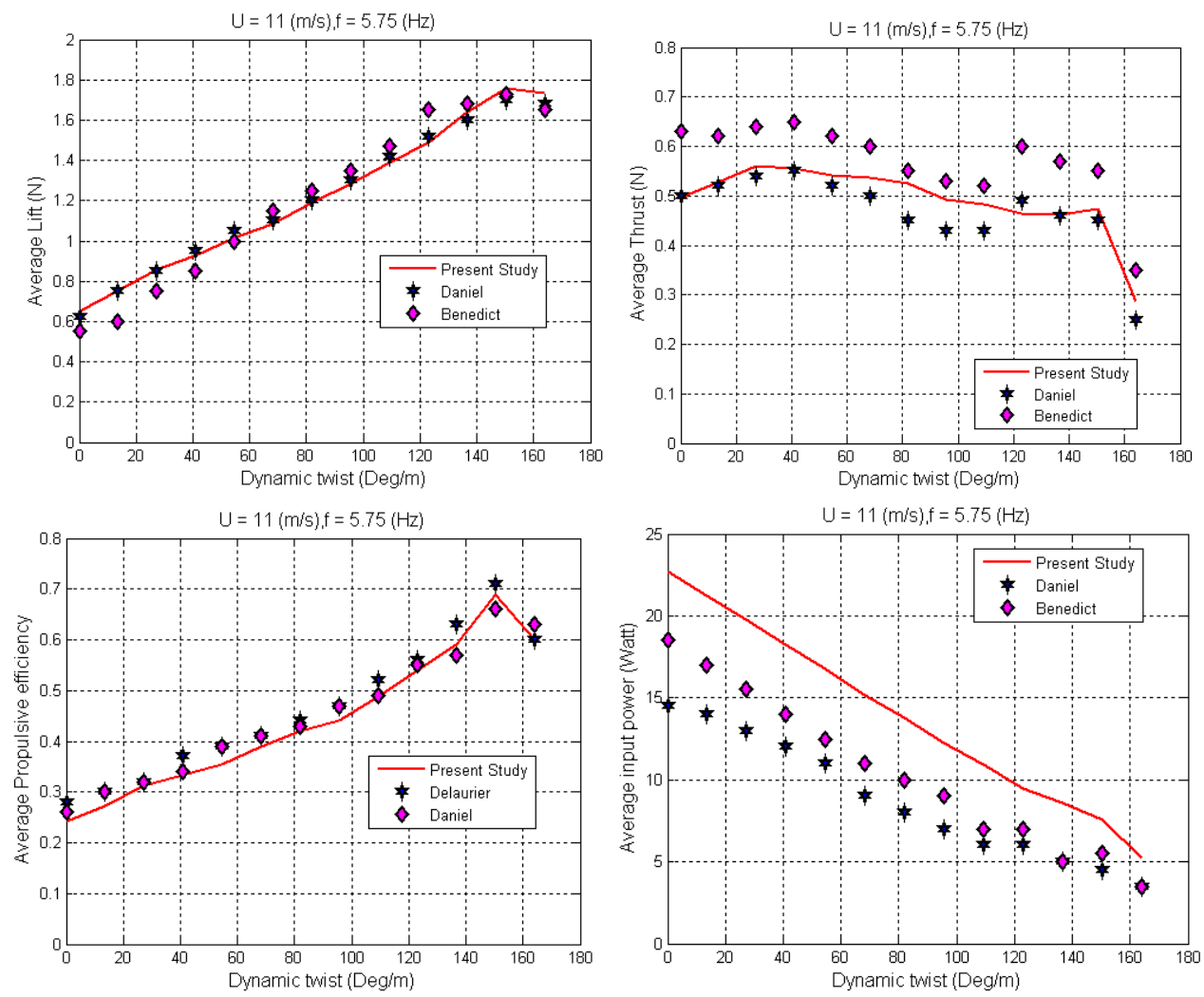

Fig. 5 Corvus monedula results
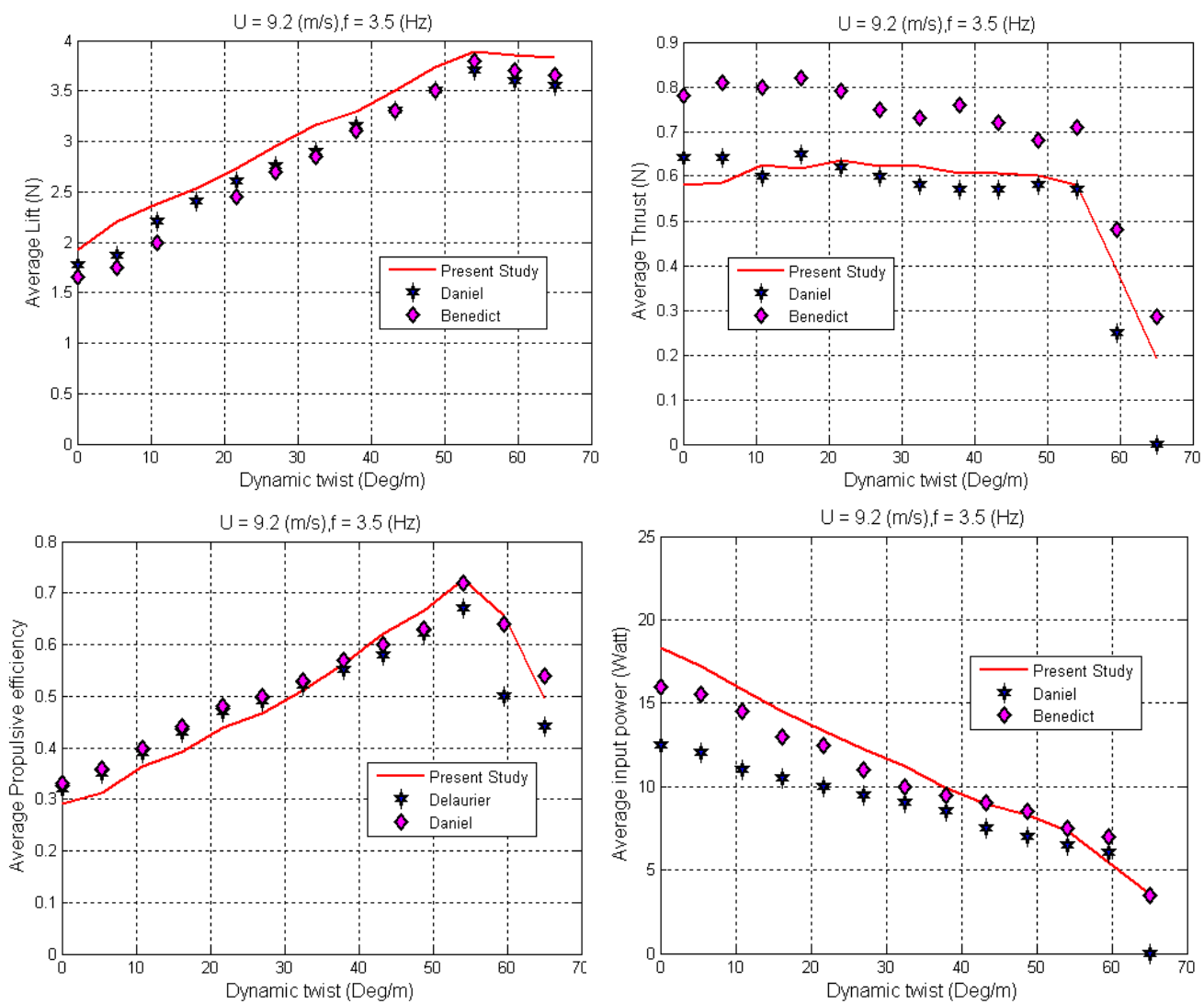

Fig. 6 Larus canas results 


\subsubsection{SlowHawk 2 flapping Model}

The ornithopter mechanism shown in Fig. 7. is chosen as a baseline prototype mechanism for this study, the shown ornithopter is the SlowHawk 2, designed and built by Sean Kinkade [9].

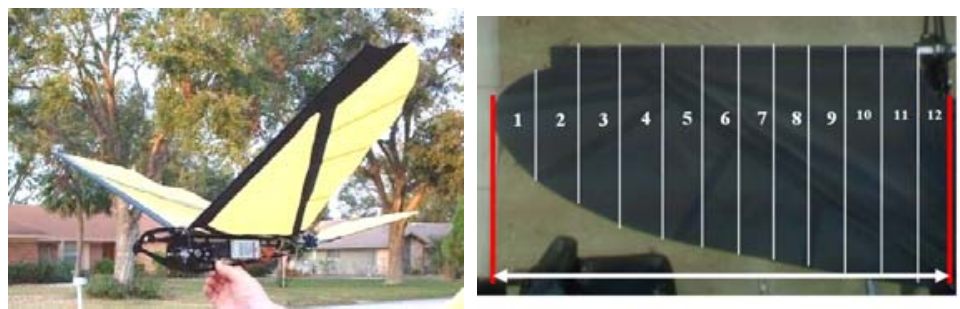

Fig. 7 The SlowHawk 2 ornithopter

The ornithopter input data is slightly similar to that of the Mew Gull, we chose the dynamic twist to be vary from $0 \div 70$ [deg/m]. A manual optimization is performed in order to ensure the best aerodynamic performance. This is performed by choosing several values of flapping frequencies with fixing all other aerodynamic variables. The computation is performed with a parametric study in order to find all the "able-to-fly" combinations of $\bar{\theta}$ and $\Gamma$. By choosing starting values to estimate the intervals for $\beta_{o}, \Gamma$ and $\bar{\theta}_{a}$. Then optimizing these values to get definite optimum operating values, based on the following requirements:

a. Average Lift condition: The major requirement is that the average Lift for a flapping cycle must be greater than or equal to the weight $W$, i.e. $(\bar{L}>W)$

b. Average propulsive efficiency condition: The average propulsive efficiency should be in the limit: $0<\bar{\eta}<1$.

The second computation search for the maximum average propulsive efficiency $\bar{\eta}_{p}$ when the above two requirements prevail. This means that for the optimized maximum average propulsive efficiency, we don't necessarily have the maximum average Lift. The computational program calculates the best combination of the dynamic twist $\beta_{o}$, maximum flapping angle magnitude $\Gamma$ and the flapping axis angle $\bar{\theta}_{a}$. For SlowHawk 2, the values assigned to some of the parameters as shown in Table 4., are used as input starting values in the optimization procedures.

Table 4 Characteristics of the SlowHawk 2

\begin{tabular}{|c|c|c|}
\hline Symbol & Value & Description \\
\hline$f$ & $2.8 \div 3.6(\mathrm{~Hz})$ & Flapping frequency range values \\
\hline$U$ & $5 \div 8(\mathrm{~m} / \mathrm{s})$ & Operating velocity range values \\
\hline$\Gamma$ & $20 \pm 10[\mathrm{deg}]$ & Flapping angle magnitude with step 5 \\
\hline
\end{tabular}

As it is clear in Fig.7., as the wing is divided into twelve strips of equal width $(d y=0.0508 m)$. Also 20 time step interval were chosen. For each strip we consider the mean chord as shown in Table 5.

Table 5 The twelve strips mean chord of for SlowHawk 2 ornithopter

\begin{tabular}{|c|c|c|c|c|c|c|c|c|c|c|c|c|}
\hline $\begin{array}{c}\text { Section } \\
\text { No. }\end{array}$ & 1 & 2 & 3 & 4 & 5 & 6 & 7 & 8 & 9 & 10 & 11 & 12 \\
\hline Chord (m) & 0.36 & 0.35 & 0.34 & 0.33 & 0.32 & 0.31 & 0.30 & 0.29 & 0.28 & 0.27 & 0.24 & 0.15 \\
\hline
\end{tabular}


Figure 8 shows, the first optimization trial for flapping frequency equals $2.8[\mathrm{~Hz}]$ and maximum flapping amplitude equals 25 [deg] with various values of $\bar{\theta}_{a}$.

Table 6., presents the optimization results obtained for the SlowHawk 2 model. All values listed in the Table 6. that corresponds to a prescribed values for $U, f, \Gamma$ and $\bar{\theta}_{a}$, where the model reaches values of average Lift which exceeds its weight $(0.42 \mathrm{~kg})$ at a minimum dynamic twist value of $30[\mathrm{deg} / \mathrm{m}]$ sufficient to be airborne. It is clear that the value in bold font does not fulfill the propulsive efficiency-requirement stated in the previous section. The values bordered by a double line give an optimum pattern for all conditions to be prevailed.
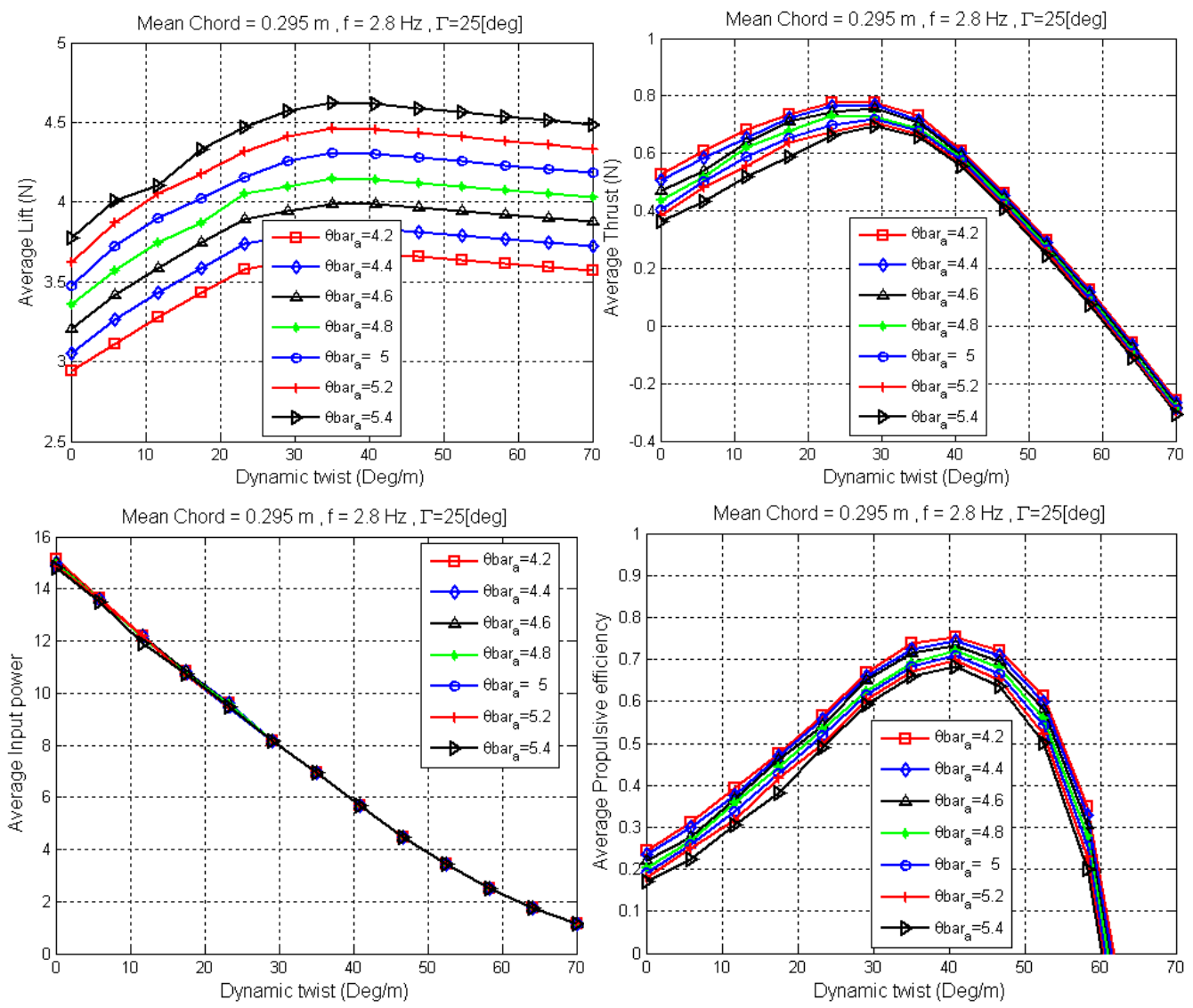

Fig. 8 SlowHawk 2 optimization results for $f=2.8[\mathrm{~Hz}], \Gamma=25[\mathrm{deg}], \mathrm{U}=7[\mathrm{~m} / \mathrm{s}]$

Table 6 Optimization results for SlowHawk 2 ornithopter

\begin{tabular}{|c|c|c|c|c|c|c|c|c|}
\hline $\begin{array}{c}\text { Opt. } \\
\text { trial }\end{array}$ & $\boldsymbol{f}[\mathbf{H z}]$ & $\bar{\theta}_{a}[\mathbf{d e g}]$ & $\Gamma[\mathbf{d e g}]$ & $\bar{\eta}[\mathbf{\%}]$ & $\bar{T}[\mathbf{N}]$ & $\bar{L}[\mathbf{N}]$ & $\bar{P}_{i n}[$ Watt] & $\beta_{o}[\mathbf{d e g} / \mathbf{m}]$ \\
\hline 1 & 2.8 & 5 & 25 & 71 & 0.7 & 4.3 & 7 & 35 \\
\hline 2 & 3 & 5 & 25 & 78 & 0.79 & 4.3 & 6.4 & 40 \\
\hline 3 & 3.2 & 5 & 25 & 88 & 0.84 & 4.35 & 6.8 & 45 \\
\hline 4 & 3.4 & 5 & 25 & 94 & 0.87 & 4.35 & 6.4 & 52 \\
\hline 5 & $\mathbf{3 . 6}$ & $\mathbf{5}$ & $\mathbf{2 5}$ & $\mathbf{1 0 4}$ & $\mathbf{1 . 0 6}$ & $\mathbf{4 . 3}$ & $\mathbf{7 . 5}$ & $\mathbf{5 2}$ \\
\hline \hline 6 & 3.4 & 4.8 & 25 & 78 & 0.62 & 4.3 & 5.2 & 35 \\
\hline 7 & $\mathbf{3 . 4}$ & $\mathbf{5}$ & $\mathbf{3 0}$ & $\mathbf{1 0 2}$ & $\mathbf{1 . 4 2}$ & $\mathbf{4 . 4}$ & $\mathbf{1 0}$ & $\mathbf{5 8}$ \\
\hline 8 & 3 & 5 & 30 & 87 & 8.5 & 4.3 & 1.08 & 52 \\
\hline
\end{tabular}


Fig.9. shows that the maximum value for propulsive efficiency is obtained for a flapping axis angle $\bar{\theta}_{a}$ equals 4.8 [deg] to be equals $78 \%$ at a dynamic twist value of 30 [deg/m] which is the same value for the ornithopter to generate sufficient Lift to overcome its weight. In the sustainable flight regime value of dynamic twist lies between 30-35 [deg/m].
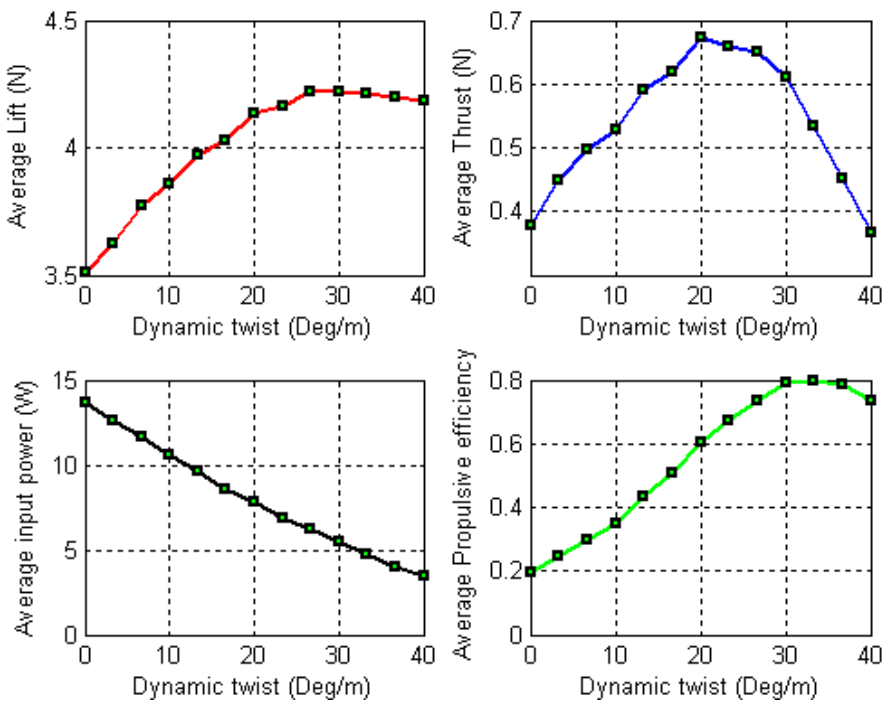

Fig. 9 SlowHawk 2 results after optimization

Fig.10. shows that while performing a modification for the stroke amplitude to be unequal, the Lift drops to lower limits, and in equal up and down strokes it fairly reaches its optimum value, which means that there is a great relation between the operating parameters like flapping angle and undisturbed velocity and operating frequency in order to reach the best performance according to Delaurier's aerodynamic model.

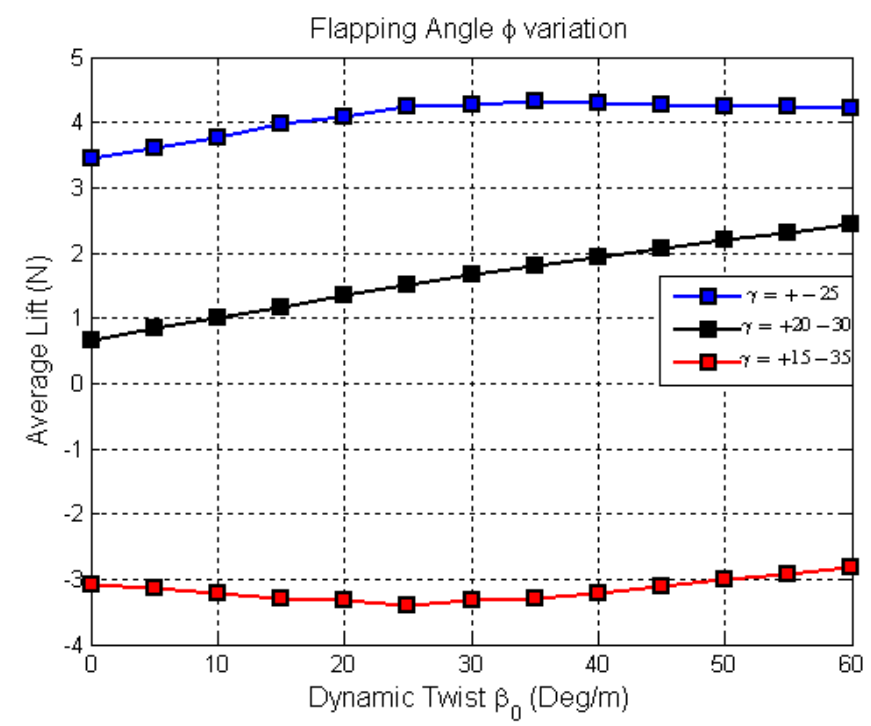

Fig. 10 Effect of flapping stroke angle variation 


\section{Conclusions}

The model presented in this work is found to produce comparable results to the one presented by Delaurier, Daniel and Benedict. Then it is used for predicting the aerodynamic characteristics of the SlowHawk 2 ornithopter assuming its wing to be rigid. The following conclusions could be arrived based on the obtain results:

- Maximum value of Lift is obtained for a specific flapping angle for all cases.

- Propulsive efficiency increases as the flapping angle increases for all cases.

- Based on dynamic twist, the propulsive efficiency achieves an optimum value for all cases.

- A long wing can have smaller twist than a shorter one and still have enough twist to generate sufficient Thrust.

- The effect of reduced frequency on the overall performance helps in determining the suitable input parameters for obtaining the optimum performance.

- The results obtained for the ornithopter is found useful for determining the experimental test parameters considering wing flexing.

The model described in this study accounts for aerodynamic effects only and it does not account for the physiological effects that might affect the optimal flapping frequency and other aerodynamic parameters.

\section{References}

[1] DeLaurier, J., “An Aerodynamic Model for Flapping-Wing Flight,” Aeronautical Journal, Vol. 97, pp. 125-130, April 1993.

[2] Ramji Kamakoti, Mats Berg, Daniel Ljungqvist and Wei Shyy. "A Computational Study of Biological Flapping Wing Flight", Transactions of the Aeronautical Society of the Republic of China, Vol 32, No (4), pages 266-279, 2000.

[3] Moble Benedict, Sudhakar K., K. Kurien Issac, "Aeroelastic Design and Manufacture of an Efficient Ornithopter Wing”, Department of Aerospace Engineering, Indian Institute of Technology, Bombay, Mumbai, June 2004.

[4] Jones, R., "The Unsteady Lift of a Wing of Finite Aspect Ratio,” NACA Report No. 681, 1940.

[5] Scherer, J.O., "Experimental and Theoretical Investigation of Large Amplitude Oscillating Foil Propulsion Systems”, Hydronautics, Laurel, Md, December 1968.

[6] Hoerner, S.F., "Skin-friction drag”, Fluid-Dynamic Drag, Published by the Author, Brick Town, NJ, pp 2-1 to 2-16, 1965.

[7] Garrick, I.E., "Propulsion of a Flapping and Oscillating Aerofoil”, NACA Report No 567, 1936.

[8] Zakaria M. Y., Bayoumy A. M., Elshabka, A. M., Abd Elhamid O.E., "Experimental Aerodynamic Characteristics of Flapping Membrane Wings ", Military Technical College, Aerospace Depatement, March 2009.

[9] Sean Kinkade, “Original Radio Controlled Ornithopter Slow Hawk 2 Instruction Manual”, HobbyTechnik, 1148 Lake Francis Drive, Apopka, Florida 32712, USA, 2008 